

\section{Arte na Pedagogia: processos educativos de poetizar, fruir e conhecer arte}




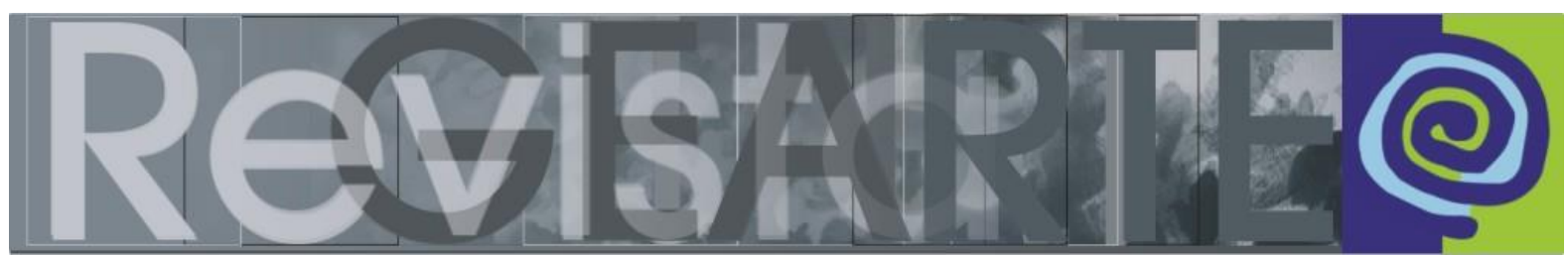

ISSN 2357-9854 | e-ISSN 2596-3198 (online)

\title{
EXPEDIENTE
}

A Revista GEARTE é um periódico quadrimestral sob a responsabilidade do Grupo de Pesquisa em Educação e Arte, do Programa de Pós-graduação em Educação da Universidade Federal do Rio Grande do Sul.

Universidade Federal do Rio Grande do Sul

Reitor: Carlos André Bulhões Mendes

Faculdade de Educação

Diretora: Liliane Ferrari Giordani

\section{Programa de Pós-graduação em Educação}

Coordenador: Sérgio Roberto Kieling Franco

\begin{abstract}
Editora-Chefe
Analice Dutra Pillar - Universidade Federal do Rio Grande do Sul (UFRGS), Porto Alegre/RS, Brasil

\section{Editoras Associadas}

Andrea Hofstaetter - Universidade Federal do Rio Grande do Sul (UFRGS), Porto Alegre/RS, Brasil Maria Helena Wagner Rossi - Universidade de Caxias do Sul (UCS), Caxias do Sul/RS, Brasil Marília Forgearini Nunes - Universidade Federal do Rio Grande do Sul (UFRGS), Porto Alegre/RS, Brasil Flávia Bastos - University of Cincinnati (UC), Cincinnati, Estados Unidos
\end{abstract}

\section{Editoras Assistentes}

Simone Rocha da Conceição - Universidade Federal do Rio Grande do Sul (UFRGS), Porto Alegre/RS, Brasil Tatiana Telch Evalte - Universidade Federal do Rio Grande do Sul (UFRGS), Porto Alegre/RS, Brasil

\section{Conselho Científico Nacional}

Ana Mae Barbosa, Universidade de São Paulo (USP), Universidade Anhembi Morumbi (UAM),São Paulo/SP, Brasil Ana Marta Meira, Universidade Federal do Rio Grande do Sul (UFRGS), Porto Alegre/RS, Brasil Anna Rita Araújo, Universidade Federal de Goiás (UFG), Goiânia/GO, Brasil

Celso Vitelli, Universidade Federal do Rio Grande do Sul (UFRGS), Porto Alegre/RS, Brasil

Consuelo Alcioni Borba Duarte Schlichta, Universidade Federal do Paraná (UFPR), Curitiba/PR, Brasil Denise Grinspum, Museus Castro Maya (MCM), Rio de Janeiro/RJ, Brasil

Fernanda Pereira da Cunha, Universidade Federal de Goiás (UFG),Goiânia/GO, Brasil

Fernando Antônio Gonçalves de Azevedo, Universidade Federal de Pernambuco (UFPE), Caruaru/ PE, Brasil Gabriela Bon, Universidade Federal do Rio Grande do Norte (UFRN), Currais Novos/RN, Brasil

Gilvânia Maurício Dias Pontes, Universidade Federal do Rio Grande do Norte (UFRN), Nata/RN, Brasil José Afonso Medeiros Souza, Universidade Federal do Pará(UFPA), Belém/PA, Brasil

Leda Maria de Barros Guimarães, Universidade Federal de Goiás (UFG),Goiânia/GO, Brasil Lívia Marques Carvalho, Universidade Federal da Paraíba (UFPB), João Pessoa/PB, Brasil

Lucia Gouvêa Pimentel, Universidade Federal de Minas Gerais(UFMG), Belo Horizonte/MG, Brasil

Lucia Teixeira de Siqueira e Oliveira, Universidade Federal Fluminense (UFF), Niterói/RJ, Brasil

Luciana Gruppelli Loponte, Universidade Federal do Rio Grande do Sul (UFRGS), Porto Alegre/RS, Brasil

Lucimar Bello Pereira Frange, Pontifícia Universidade Católica de São Paulo (PUCSP), São Paulo/SP, Brasil

Marcos Villela Pereira, Pontifícia Universidade Católica do Rio Grande do Sul (PUCRS), Porto Alegre/ RS, Brasil Maria Christina Rizzi, Universidade de São Paulo (USP), São Paulo - SP, Brasil

Maria Isabel Petry Kehrwald, Fundação Municipal de Artes de Montenegro (FUNDARTE), Montenegro/RS, Brasil

Maria Lúcia Batezat Duarte, Universidade do Estado de Santa Catarina (UDESC), Florianópolis/SC, Brasil

Marion Divério Faria Pozzi, Universidade Federal do Rio Grande do Sul (UFRGS), Porto Alegre/RS, Brasil

Mirian Celeste Martins, Universidade Presbiteriana Mackenzie (MACKENZIE), São Paulo/SP, Brasil

Moema Lúcia Martins Rebouças, Universidade Federal do Espírito Santo (UFES), Vitória/ES, Brasil

Nadja de Carvalho Lamas, Universidade da Região de Joinville (UNIVILLE), Joinville/SC, Brasil 


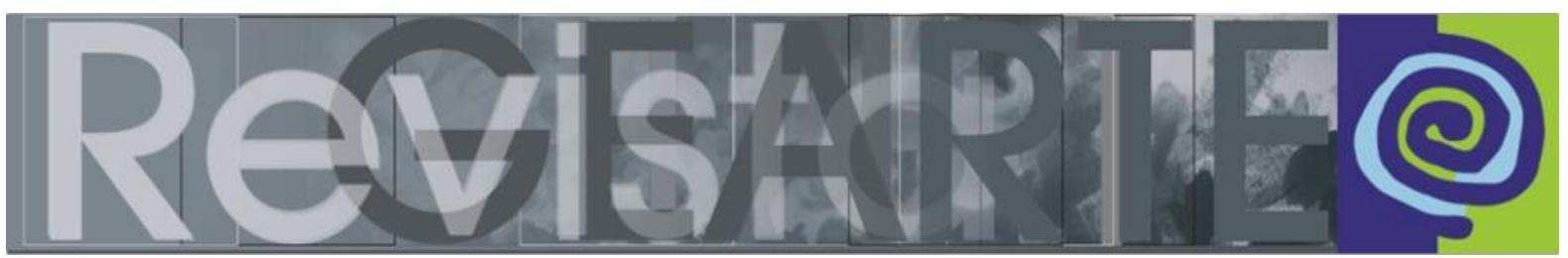

Regina Maria Varini Mutti, Universidade Federal do Rio Grande do Sul (UFRGS), Porto Alegre/RS, Brasil Rejane Reckziegel Ledur, Secretaria Municipal de Educação de Canoas (SME), Canoas/RS, Brasil

Rita Inês Petrykowski Peixe, Instituto Federal de Santa Catarina (IFSC), Itajaí/SC, Brasil

Rita Luciana Berti Bredariolli, Universidade Estadual Paulistana Júlio de Mesquita (UNESP), São Paulo/SP, Brasil Rosa lavelberg, Universidade de São Paulo (USP), São Paulo /SP, Brasil

Rosana Fachel de Medeiros, Secretaria Municipal de Educação de Canoas (SME), Canoas/RS, Brasil Rosângela Fachel de Medeiros, Universidade Regional Integrada do Alto Uruguai e das Missões (URI), Frederico Westphalen/RS, Brasil

Ruth Rejane Perleberg Lerm, Instituto Federal de Educação, Ciência e Tecnologia Sul-rio-grandense (IFSul), Pelotas/RS, Brasil

Sandra Regina Ramalho e Oliveira, Universidade do Estado de Santa Catarina (UDESC), Florianópolis/SC, Brasil Silvia Sell Duarte Pillotto, Universidade da Região de Joinville (UNIVILLE), Joinville/SC, Brasil

Solange de Fátima Gabre, Secretaria Municipal de Educação de Curitiba (SME), Curitiba/PR, Brasil Umbelina Duarte Barreto, Universidade Federal do Rio Grande do Sul (UFRGS), Porto Alegre/RS, Brasil

\section{Conselho Científico Internacional}

Fernando Hernández, Universidad de Barcelona, Espanha

Imanol Aguirre Arriaga, Universidad Pública de Navarra, Espanha José Carlos de Paiva, Universidade do Porto, Portugal

Justin P. Sutters, George Mason University, Estados Unidos da América do Norte Lourenço Eugênio Cossa, Universidade Pedagógica, Moçambique

María Acaso López-Bosch, Universidad Complutense de Madrid, Espanha

Michael Parsons, University of Illinois, Estados Unidos da América do Norte Norman Freeman, University of Bristol, Reino Unido

Olga Lucia Olaya Parra, Academia Superior de Artes de Bogotá, Colômbia

Raquel Ribeiro dos Santos, Fundação Caixa Geral de Depósito, Portugal

Ricardo Marín-Viadel, Universidad de Granada, Espanha

Ricardo Rubiales García Jurado, Consejo Estatal para la Cultura y las Artes, México Teresa Torres Eça, Universidade do Porto, Portugal

Revisora

Liane Batistela Kist - Centro Universitário Franciscano (UNIFRA), Santa Maria/RS, Brasil

\section{Diagramador}

Edson Leonel de Oliveira - Universidade Federal do Rio Grande do Sul (UFRGS), Porto Alegre/RS, Brasil

Bolsista

Bianca Bottini - Universidade Federal do Rio Grande do Sul (UFRGS), Porto Alegre/RS, Brasil

\section{Capa}

Umbelina Barreto - Universidade Federal do Rio Grande do Sul (UFRGS), Porto Alegre/RS, Brasil

\section{Apoio}

O presente trabalho foi realizado com apoio da Coordenação de Aperfeiçoamento de Pessoal de Nível Superior - Brasil (CAPES) - Código de Financiamento 001

e do Programa de Apoio à Edição de Periódicos PAEP / UFRGS

\section{Contatos}

Universidade Federal do Rio Grande do Sul

Programa de Pós-Graduação em Educação

Grupo de Pesquisa em Educação e Arte - GEARTE

Av. Paulo Gama, s/no, prédio 12201, sala 727 - Centro, CEP 90046-900, Porto Alegre/RS

Revista: http://www.seer.ufrgs.br/gearte

Site do grupo: http://www.ufrgs.br/gearte

Telefone: (51) 3308-4145

E-mail: gearte.ufrgs@gmail.com 